\title{
Cytotoxic responses of human chondrocytes to bupivacaine, levobupivacaine, and ropivacaine
}

\author{
Pudkrong Kaewpichit', Somrat Charuluxananan², Monpichar Srisa-Art ${ }^{3}$, Sarocha Sisawat ${ }^{2}$, \\ Vitavat Aksornkitti ${ }^{4}$, Chalermsri Chayudsatid ${ }^{4}$, Amornpun Sereemaspun ${ }^{4, *}$
}

\section{Abstract}

Background: Intra-articular injections of local anesthetics are used commonly in articular surgery. However, chondrocyte viability and metabolism may be adversely affected by various anesthetics.

Objectives: To assess the chondrotoxic effects of bupivacaine, levobupivacaine, and ropivacaine on human chondrocytes and elucidate possible mechanisms of chondrocyte death.

Methods: Cultured human chondrocytes $(\mathrm{CHON}-001)$ were exposed to $0.25 \%$ or $0.5 \%$ of bupivacaine, levobupivacaine, and ropivacaine in vitro. Cell viability was determined by flow cytometry after 15, 30, 60, and 120 min of exposure. Chondrocyte reactive oxygen species (ROS) production was measured every $10 \mathrm{~min}$ for up to $1 \mathrm{~h}$ using $2^{\prime}, 7^{\prime}$ dichlorodihydrofluorescein staining. Chondrocyte production of glycosaminoglycan was measured by capillary electrophoresis. NO production was measured using a colorimetric assay kit.

Results: We found a significant increase in chondrotoxicity dependent on exposure time and concentration of the anesthetic. At $60 \mathrm{~min}$, chondrocyte viability was significantly $(P<0.05)$ decreased when exposed to $0.5 \%$ levobupivacaine $(32.5 \%)$, or $0.25 \%$ or $0.5 \%$ bupivacaine $(34.3 \%$ or $46.5 \%$, respectively) compared with exposure to phosphate-buffered saline (PBS) vehicle as a control. Cell death at $120 \mathrm{~min}$ was mainly necrosis. There was no difference in viability after treatment with either concentration $(0.25 \%$ or $0.5 \%)$ of ropivacaine at any time compared with exposure to PBS. We found increased production of NO, while ROS decreased after exposure to any of the anesthetics tested.

Conclusions: Ropivacaine may be safer than bupivacaine or levobupivacaine as an intra-articular analgesic. Chondrotoxicity of anesthetics in vitro may be mediated via a reactive nitrogen species-dependent pathway.

Keywords: bupivacaine; glycosaminoglycans; levobupivacaine; reactive oxygen species; ropivacaine

In outpatient settings for orthopedics, local anesthetics are commonly injected intra-articularly. Such treatment is commonly considered as part of a multimodal regimen for postoperative analgesia following procedures involving joints such as the shoulder, knee, hip, and ankle $[1,2]$. Although it provides effective pain control, intra-articular administration

*Correspondence to: Amornpun Sereemaspun, Department of Anatomy, Faculty of Medicine, Chulalongkorn University, Bangkok 10330,

Thailand, e-mail: amornpun.s@chula.ac.th

'Department of Anesthesiology, Faculty of Medicine, Chulalongkorn University, King Chulalongkorn Memorial Hospital, Thai Red Cross, Bangkok 10330, Thailand

2Department of Anesthesiology, Faculty of Medicine, Chulalongkorn University, Bangkok 10330, Thailand

${ }^{3}$ Department of Chemistry, Faculty of Science, Chulalongkorn University, Bangkok 10330, Thailand

${ }^{4}$ Nanomedicine Research Unit, Department of Anatomy, Faculty of Medicine, Chulalongkorn University, Bangkok 10330, Thailand

"These authors contributed equally to first authorship of this article.

כ Open Access. ๑ 2018 Pudkrong Kaewpichit et al., published by Sciendo. (cc)BY-NC-ND This work is licensed under the Creative Commons Attribution NonCommercial-NoDerivatives 4.0 License. 
of local anesthetics has attracted attention for its potentially deleterious effects on articular chondrocytes. Studies in vitro have demonstrated that local anesthetics can induce cell death in both animal and human chondrocytes [3-5]. Clinical evidence for chondrotoxicity of local anesthetics in humans has been reported, and there are an increasing number of case reports implicating association between intra-articular injections to manage pain and the development of chondrolysis, a catastrophic development of progressive pain and stiffness of joints $[1,2,6]$. This evidence suggests that exposure to local anesthetics might be potentially toxic for chondrocytes. However, the cellular mechanism for this cytotoxicity has not been fully elucidated.

The purpose of the present study was to examine human articular chondrocyte viability and glycosaminoglycan (GAG) production after exposure to various concentrations of bupivacaine, levobupivacaine, and ropivacaine for various times in vitro. We also sought to determine whether the cytotoxicity observed involved the production of reactive oxygen species (ROS) or nitric oxide. We hypothesized that chondrotoxicity was directly dependent on the concentration of local anesthetics, exposure time, and type of local anesthetic.

\section{Materials and methods}

\section{Cell culture}

The human chondrocyte cell line (CHON-001) was purchased from the American Type Culture Collection (and had been previously authenticated for identity by karyotyping and short tandem repeat profile) and cultured in Dulbecco's modified Eagle's medium (DMEM), supplemented with 10\% fetal bovine serum and $1 \%$ antibiotic-antimycotic solution (Gibco), but was not recently tested for mycoplasma contamination. The cells were cultured in Flask T75 (Nest Biotechnology, China) and incubated at $37^{\circ} \mathrm{C}$ under an atmosphere of $5 \%$ $\mathrm{CO}_{2}$ until used (the passages of CHON-001 used in the present study ranged from 5-10).

\section{Cellular viability, morphology, and death pattern analysis}

The cytotoxicity of the anesthetics on chondrocytes and pattern of cell death were analyzed using an Epics XL flow cytometer (Beckman Coulter). To conduct this analysis, CHON-001 cells were seeded into 24-well plates at a final concentration of $2.5 \times 10^{5}$ cells/well and incubated overnight at $37^{\circ} \mathrm{C}$ under an atmosphere of $5 \% \mathrm{CO}_{2}$. Before treatment with anesthetics, the cells were washed using phosphate-buffered saline (PBS). The local anesthetics, bupivacaine, levobupivacaine, and ropivacaine at low dose $(0.25 \%)$ and high dose $(0.5 \%)$ were applied to the cells, using PBS alone as a negative control and $\mathrm{H}_{2} \mathrm{O}_{2}$ as a positive control. Then, the cells in each treatment were incubated at $37^{\circ} \mathrm{C}$ under an atmosphere of $5 \% \mathrm{CO}_{2}$ for 30,60 , and $120 \mathrm{~min}$. Three replicates were used for each treatment and time. After incubation, cells were collected from two parts of the well plates, (i) cells that detached from surface of plate and were suspended in culture media, and (ii) cells from plate surface, which were collected using trypsin-EDTA (Gibco), and combined with the planktonic cells. The cells collected were examined under a microscope at $10 \times$ magnification then stained with annexin $\mathrm{V}$-conjugated fluorescein isothiocyanate (FITC) and propidium iodide (PI; BD biosciences) according to the manufacturer's instructions. Briefly, $\mathrm{CHON}-001$ cells were washed twice with cold PBS and resuspended in annexin V-binding buffer. The FITC-conjugated annexin V and PI were added into the cell-collection tube. The cells were incubated in the dark at room temperature (about $25^{\circ} \mathrm{C}$ ) for $15 \mathrm{~min}$. After staining, and flow cytometry the data were analyzed using system II software (version 3.0; Beckman Coulter).

\section{GAG detection}

\section{Chemicals and reagents}

Dermatan sulfate (sodium salt) and sodium hyaluronic acid from cock's comb were obtained from TCI Europe. Chondroitin sulfate (sodium salt) from shark cartilage, butylamine and (2-hydroxypropyl)- $\beta$-cyclodextrin were purchased from Sigma-Aldrich. Sodium dihydrogen phosphate, methanol, phosphoric acid, and sodium hydroxide were reagent grade.

\section{Instrumentation and conditions}

Capillary electrophoresis analyses were performed on a Beckman Coulter P/ACE MDQ capillary electrophoresis system equipped with a photodiode array detector operating at $195 \mathrm{~nm}$. Separation and analysis were conducted using an uncoated fused silica capillary (50 $\mu \mathrm{m}$ internal diameter, $375 \mu \mathrm{m}$ outside diameter) with a total length of $40.2 \mathrm{~cm}$ (30 $\mathrm{cm}$ to detector). Samples were introduced into the capillary using pressure injection ( $0.5 \mathrm{psi}$ for $60 \mathrm{~s})$ and separated at $-16 \mathrm{kV}$. The temperature of the capillary was regulated by liquid cooling at $25^{\circ} \mathrm{C}$. A new capillary was first conditioned using solutions as follows: methanol, $1 \mathrm{M} \mathrm{NaOH}, 0.1 \mathrm{M}$ $\mathrm{NaOH}$, Milli-Q water, $1 \mathrm{M} \mathrm{H}_{3} \mathrm{PO}_{4}$, and $0.1 \mathrm{M} \mathrm{H}_{3} \mathrm{PO}_{4}$, followed by the background electrolyte. Each solution was flushed 
into the capillary using 20 psi for $20 \mathrm{~min}$. Between runs, the capillary was rinsed with methanol for $1 \mathrm{~min}, 0.1 \mathrm{M} \mathrm{H}_{3} \mathrm{PO}_{4}$ for $1 \mathrm{~min}$, and the background electrolyte for $2 \mathrm{~min}$. Electropherograms were acquired and analyzed using 32 Karat software (version 5.0). Before capillary electrophoresis runs, all samples were stored at $4^{\circ} \mathrm{C}$.

\section{Preparation of reagents and samples}

The background electrolyte was prepared by dissolving appropriate amount of $\mathrm{NaH}_{2} \mathrm{PO}_{4}$ and (2-hydroxypropyl)- $\beta$ cyclodextrin into an optimized volume of butylamine, and adjusted to $\mathrm{pH} 4.0$ with $1 \mathrm{M} \mathrm{H}_{3} \mathrm{PO}_{4}$ and $1 \mathrm{M} \mathrm{NaOH}$ to give the optimized conditions indicated below. A standard stock solution of $1,000 \mathrm{mg} \cdot \mathrm{L}^{-1}$ of chondroitin sulfate, dermatan sulfate, and hyaluronic acid was prepared separately using Milli-Q water. The standard working solutions were prepared by diluting from the standard stock solutions.

CHON-001 cells were seeded into 12-well plates at a final concentration of $2 \times 10^{5}$ cells/well and incubated overnight at $37^{\circ} \mathrm{C}$ under an atmosphere of $5 \% \mathrm{CO}_{2}$. Before cell treatment, bupivacaine was dispersed in DMEM at a final concentration of $0.25 \%$ and $0.125 \%$. The cells were exposed to this anesthetic for $24 \mathrm{~h}$; then, supernatants were collected to determine the amount of the GAGs produced including dermatan sulfate, chondroitin sulfate, and hyaluronic acid. Briefly, supernatant from each condition was filtered through $0.22 \mu \mathrm{m}$ nylon membrane filters and analyzed by capillary electrophoresis using the following optimized conditions: $200 \mathrm{mM} \mathrm{NaH}_{2} \mathrm{PO}_{4}$, $200 \mathrm{mM}$ butylamine, $80 \mathrm{mM}$ (2-hydroxypropyl)- $\beta$-cyclodextrin, separation voltage of $-16 \mathrm{kV}$, injection time of $60 \mathrm{~s}$, and ultraviolet detection at $195 \mathrm{~nm}$.

\section{ROS production}

Generated ROS were determined by $2^{\prime}, 7^{\prime}$-dichlorodihydrofluorescein diacetate (DCFH) reagent (Invitrogen). In brief, CHON-001 cells were seeded into black 96-well plates at confluent density of 10,000 cells/well and then incubated at $37^{\circ} \mathrm{C}$ under an atmosphere of $5 \% \mathrm{CO}_{2}$ overnight. The cells were treated with DCFH and incubated in the dark for $30 \mathrm{~min}$. After incubation was complete, the cells were treated with anesthetic under the same conditions as for the cytotoxicity experiment previously described. When nonfluorescent DCFH penetrates the cells, intracellular esterases hydrolyze DCFH to 2',7'-dichlorodihydrofluorescein that is oxidized by intracellular ROS to $2^{\prime}, 7^{\prime}$-dichlorofluorescein, which can be detected by fluorescence emission at $528 \mathrm{~nm}$ using a microplate reading fluorescence spectrophotometer at an excitation of $485 \mathrm{~nm}$. The intensity of the fluorescence in each condition was measured every $10 \mathrm{~min}$ for $1 \mathrm{~h}$ to assess the kinetic change of ROS production by cells when exposed to anesthetics.

\section{Nitric oxide production}

Nitric oxide (NO) production was measured by using a colorimetric Nitrite/Nitrate Assay kit (Sigma-Aldrich) according to the instructions from the manufacturer. In brief, CHON-001 cells were seeded into 24 -well plates at a final concentration of $6 \times 10^{4}$ cells and incubated overnight. The cells were treated with anesthetic at the same concentration as for the cell viability analysis. Then, the CHON-001 cells were incubated for 15 , 30 , and $120 \mathrm{~min}$ before collecting supernatant to measure their NO production. Supernatants were collected from 24-well plates into 96-well plates and incubated with nitrate reductase and enzyme cofactor solution for $2 \mathrm{~h}$. After incubation, Griess reagents $\mathrm{A}$ and $\mathrm{B}$ were added to each well and incubated with the contents of the wells for $10 \mathrm{~min}$. The absorbance of the well contents was measured at $540 \mathrm{~nm}$ using a microplate reading spectrophotometer. Finally, the absorbance of a blank solution was subtracted from the absorbance of each well contents and the concentration of nitrite-nitrate was determined from a calibration curve.

\section{Statistical analyses}

Data are presented as mean values and standard deviation (mean \pm SD) calculated using GraphPad Prism software (version 5.0) for the outcome variables of cell viability, GAG synthesis, ROS, and NO generation. One way analysis of variance, with a Tukey post hoc multiple comparison test was used to compare the local anesthetics and control groups. $P<0.05$ was assumed significant in tests of statistical inference. The results for each treatment and time were determined in triplicate, except GAG detection, which was not repeated because of limitations in using and availability of the instrument.

\section{Results}

\section{Cytotoxicity}

Cell viability decreased in a time- and concentration-dependent manner after treatment with the various types of local anesthetics. Chondrocyte viability was reduced significantly after exposure to levobupivacaine and bupivacaine (Figure 1). Compared with treatment with PBS vehicle control, we found no significant effect of $0.25 \%$ levobupivacaine, or $0.25 \%$ or 


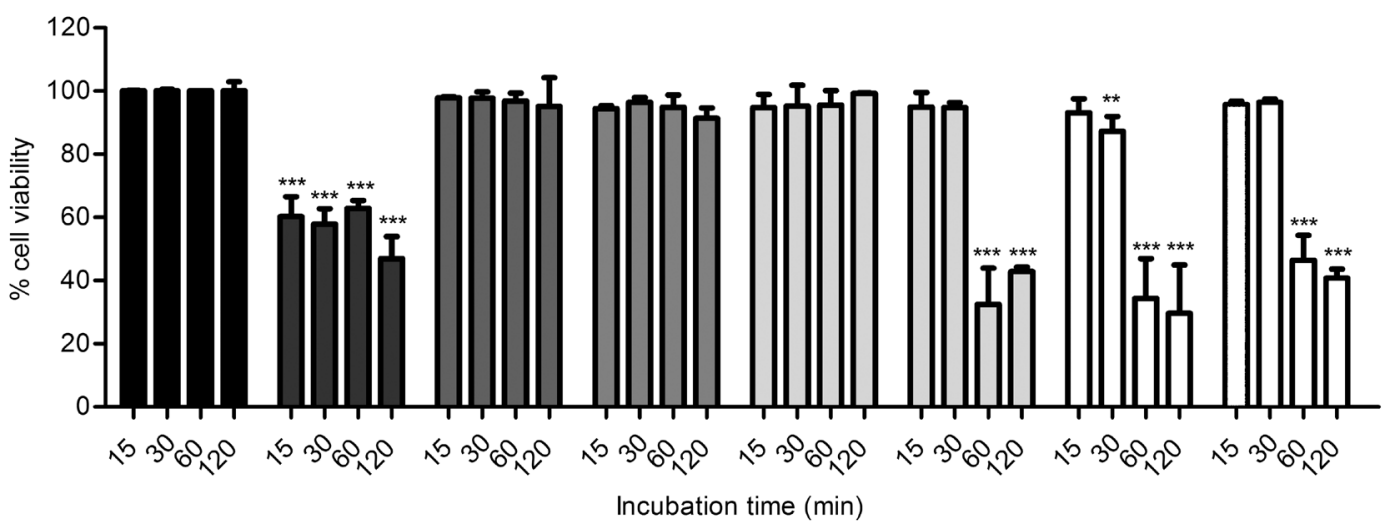

Figure 1. Effect of local anesthetics on viability of CHON-001 cells after 15-, 30-, 60-, and 120-min exposure as determined by FITC-conjugated annexin V and PI staining and cytometry using a Beckman Coulter Epics XL flow cytometer. The sets of bars represent the mean percentages of chondrocyte viability after treatment with local anesthetic or controls; from left to right, PBS vehicle control (black bars), $0.4 \mathrm{mM} \mathrm{H}_{2} \mathrm{O}_{2}$ positive control (dark-gray bars), $0.25 \%$ ropivacaine (medium-gray bars), $0.5 \%$ ropivacaine (medium-gray bars), $0.25 \%$ levobupivacaine (light-gray bars), $0.5 \%$ levobupivacaine (light-gray bars), $0.25 \%$ bupivacaine (white bars), $0.5 \%$ bupivacaine (white bars). Low $(0.25 \%)$ and high ( $0.5 \%)$ concentrations of bupivacaine and $0.5 \%$ levobupivacaine treated groups significantly decreased cell viability at 60 min, compared with treatment by the PBS vehicle control. ${ }^{* *} P<0.001,{ }^{* * *} P<0.001$; error bars represent standard deviation; FITC, fluorescein isothiocyanate; PBS, phosphate-buffered saline; $\mathrm{PI}$, propidium iodide

$0.5 \%$ ropivacaine on cell viability. At $60 \mathrm{~min}$, viability was decreased significantly in chondrocytes treated with $0.5 \%$ levobupivacaine $(32.5 \pm 11.5 \%)$, or $0.25 \%$ or $0.5 \%$ bupivacaine $(34.2 \pm 12.6 \%$ and $46.5 \pm 7.9 \%$, respectively) compared with chondrocytes treated with PBS vehicle control. Viability was decreased significantly $(87.3 \pm 4.7 \%, P<0.001)$ in chondrocytes treated with $0.25 \%$ bupivacaine at 30 min compared with chondrocytes treated with PBS vehicle as a control.

\section{Cell death pattern analysis}

The pattern of chondrocyte death was mainly necrosis after exposure to $0.25 \%$ bupivacaine $(69.1 \%), 0.5 \%$ bupivacaine $(56.0 \%)$, or $0.5 \%$ levobupivacaine $(51.0 \%)$ for $120 \mathrm{~min}$ (Figure 2). The chondrocyte morphology after treatment with low- or high-dose bupivacaine changed from round to elongated, compared with chondrocytes treated with PBS as a control.

\section{GAG detection}

The amount of dermatan sulfate in chondrocytes treated with DMEM for $24 \mathrm{~h}$ was $3784 \mathrm{AU}$, treated similarly with $\mathrm{H}_{2} \mathrm{O}_{2}$ was $14649 \mathrm{AU}, 0.125 \%$ bupivacaine was $19344 \mathrm{AU}$, and $0.25 \%$ bupivacaine was 9771 AU. Compared with DMEM as a negative control, $0.125 \%$ bupivacaine significantly increased the amount of dermatan sulfate in chondrocytes (Figure 3A and B), while the levels of neither chondroitin sulfate nor hyaluronic acid changed significantly after exposure to bupivacaine.

\section{ROS production}

The levels of ROS were decreased significantly in chondrocytes treated with bupivacaine, levobupivacaine, or ropivacaine, and low concentrations were apparently more effective than high concentration for all local anesthetics (Figure 4A-F). At $60 \mathrm{~min}$, ROS production was significantly lower in the chondrocytes treated with $0.25 \%$ bupivacaine $(54.0 \pm 3.9, P<0.05)$, $0.25 \%$ levobupivacaine $(61.05 \pm 0.62, P<0.01)$, or $0.25 \%$ ropivacaine $(70.7 \pm 1.8 \%, P<0.001)$ compared with that after treatment with PBS vehicle as a control. ROS production by chondrocytes after treatment with $0.5 \%$ bupivacaine $(82.9 \pm$ $12.0 \%, P<0.05), 0.5 \%$ levobupivacaine $(70.0 \pm 5.1 \%, P<$ $0.01)$, or $0.5 \%$ ropivacaine $(69.8 \pm 5.5 \%, P<0.001)$ was also significantly lower than that after treatment with PBS vehicle as a control.

\section{NO production}

NO production by chondrocytes treated with local anesthetics was significantly higher than by vehicle control treated chondrocytes (Figure 5). At $120 \mathrm{~min}$, the NO production was highest in chondrocytes treated with $0.25 \%$ levobupivacaine (173.6\%, $P<0.001)$ or $0.5 \%$ levobupivacaine $(215.8 \%, P<$ $0.001)$. NO production was also high in chondrocytes treated with $0.25 \%$ ropivacaine $(164.0 \%, P<0.01), 0.5 \%$ ropivacaine (198.1\%, $P<0.01$ ), or $0.25 \%$ bupivacaine $(123.2 \% P<0.05)$, but low in the group treated with $0.5 \%$ bupivacaine $(83.7 \% P$ $<0.05)$. 
A

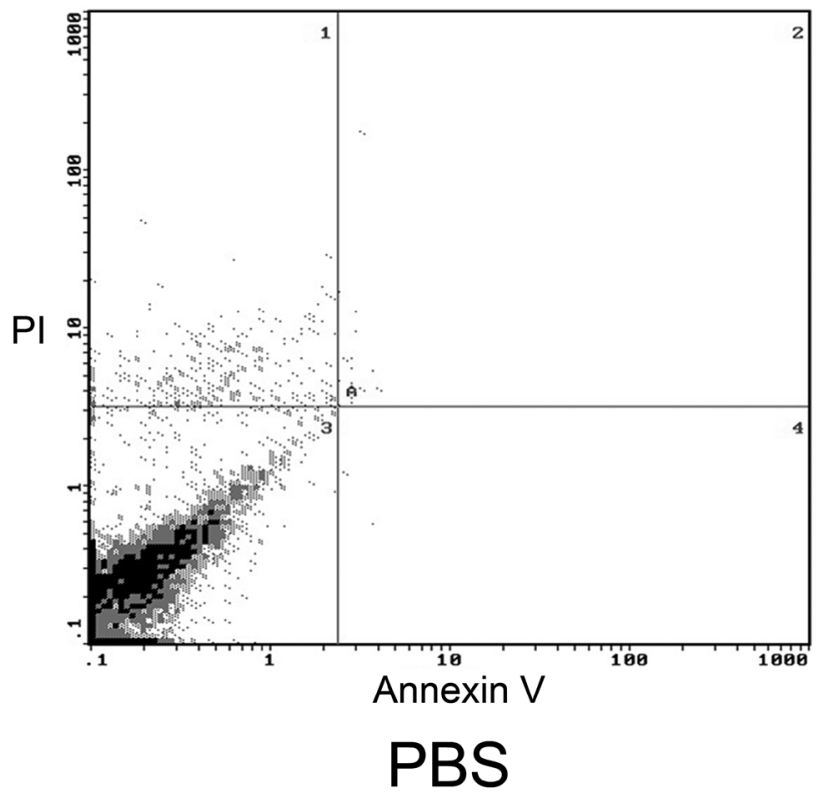

\section{C}

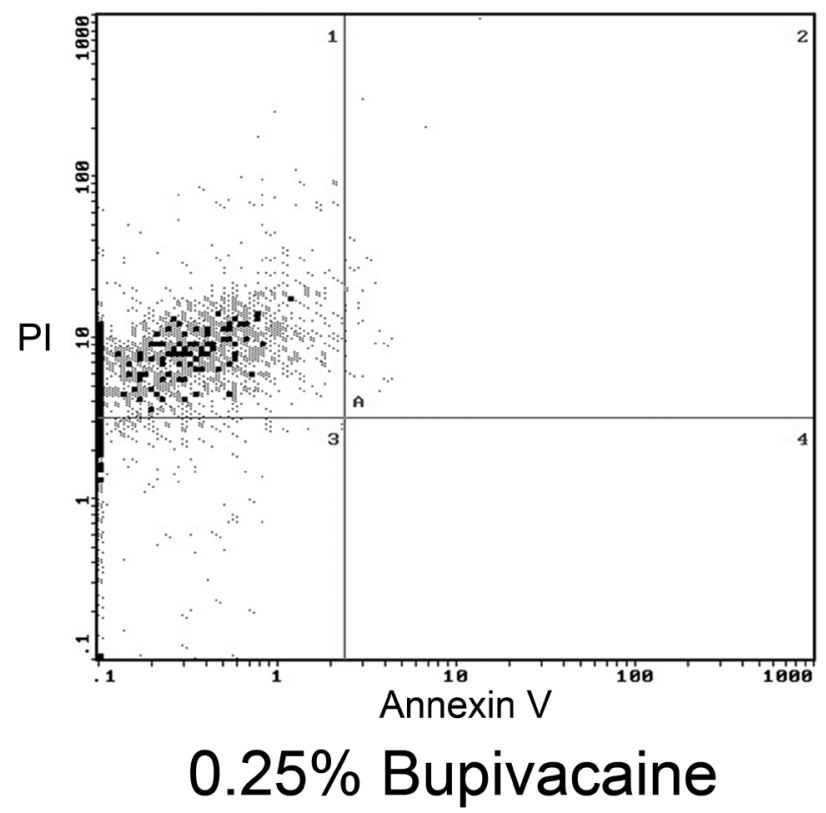

B

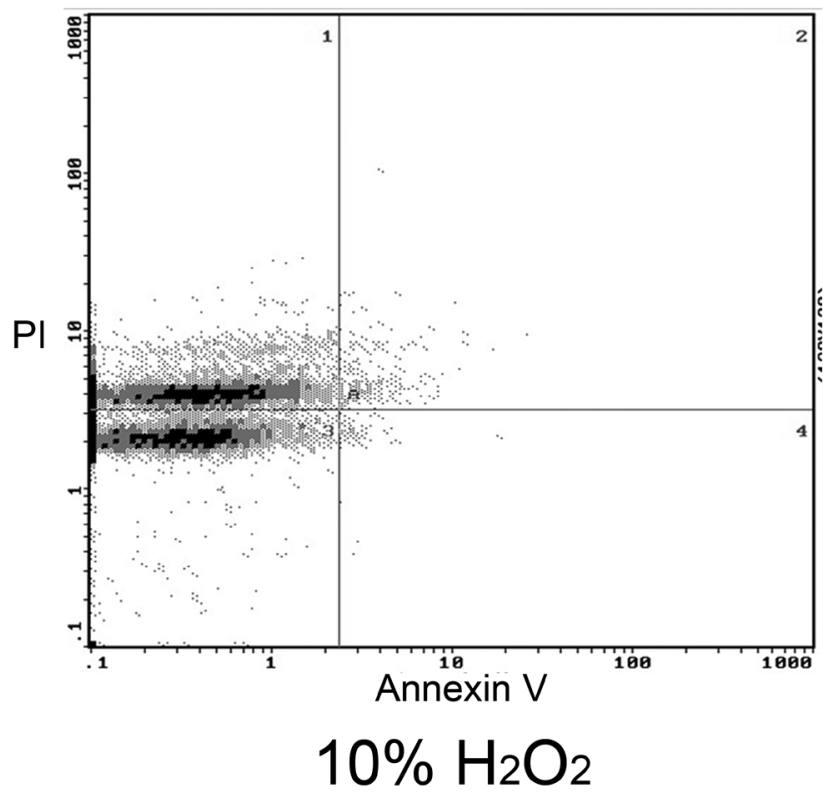

D

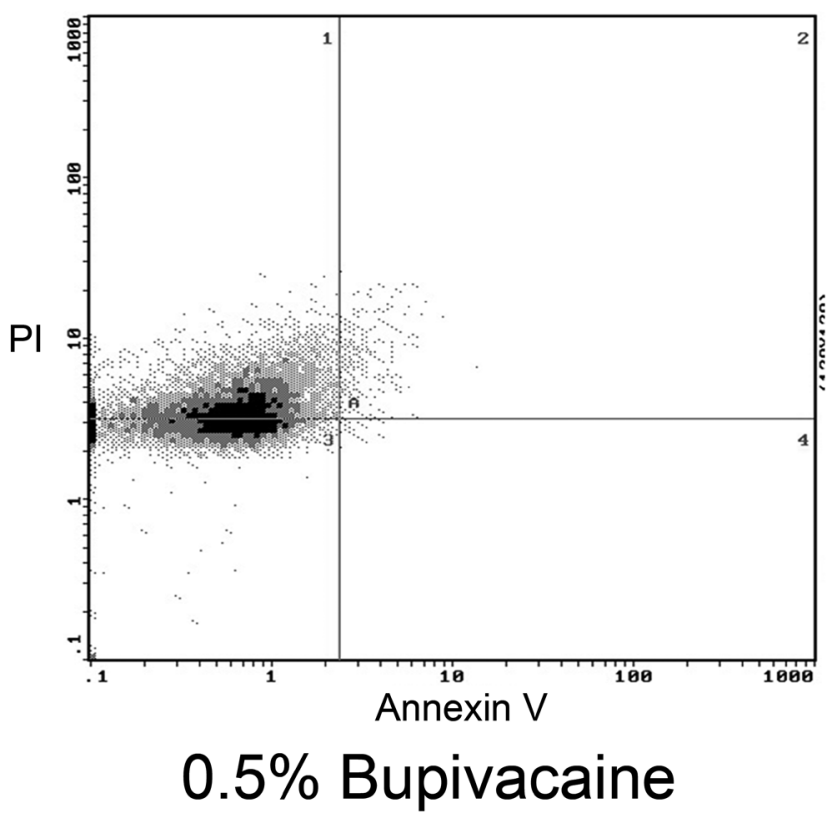

Figure 2A-D. Pattern of CHON-001 cell death after 120 min exposure to local anesthetics as analyzed using a Beckman Coulter Epics XL flow cytometer after staining with annexin V-conjugated FITC and PI. Compared with chondrocytes treated with PBS vehicle as a control (A), the numbers of necrotic chondrocytes increased after treatment with $0.25 \%$ bupivacaine (C), $0.5 \%$ bupivacaine (D), $0.4 \mathrm{mM} \mathrm{H}_{2} \mathrm{O}_{2}$ positive control (B) FITC, fluorescein isothiocyanate; PBS, phosphate-buffered saline; PI, propidium iodide

\section{Discussion}

In the present study, we examined some cellular responses of human chondrocytes after exposure to local anesthetics. We found that bupivacaine and levobupivacaine were chondrotoxic, while ropivacaine was not. The chondrotoxic effect was time, concentration, and drug-type dependent. These findings suggest that ropivacaine may be safer than bupivacaine or levobupivacaine as an intra-articular analgesic. 
E

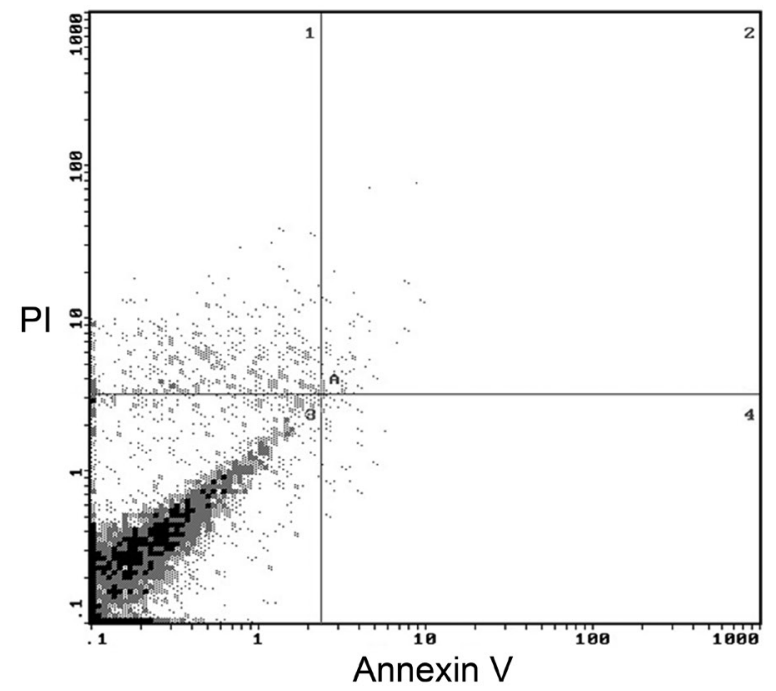

$0.25 \%$ Levobupivacaine

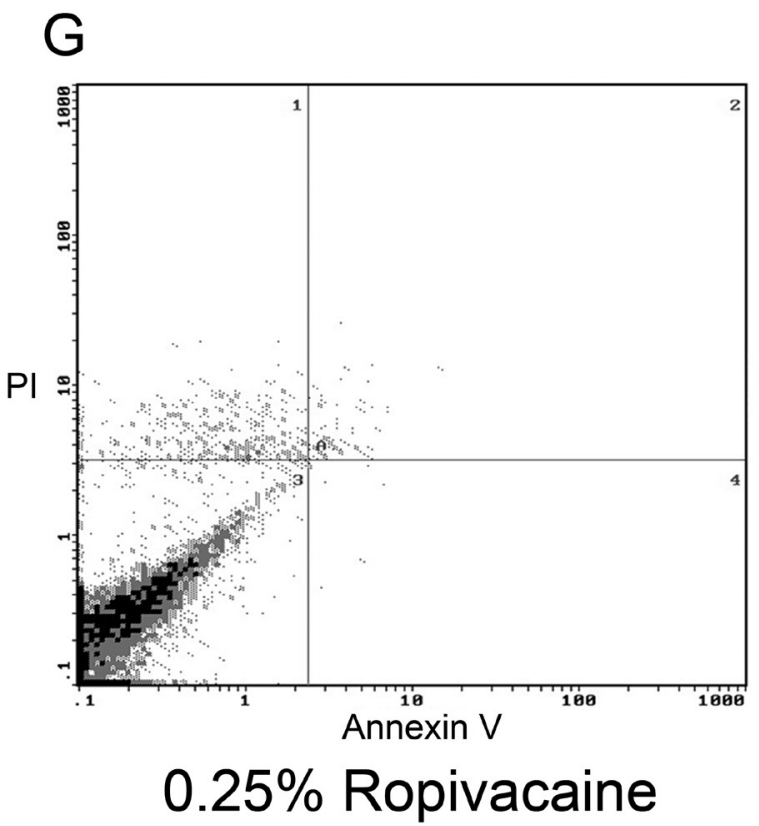

$\mathrm{F}$
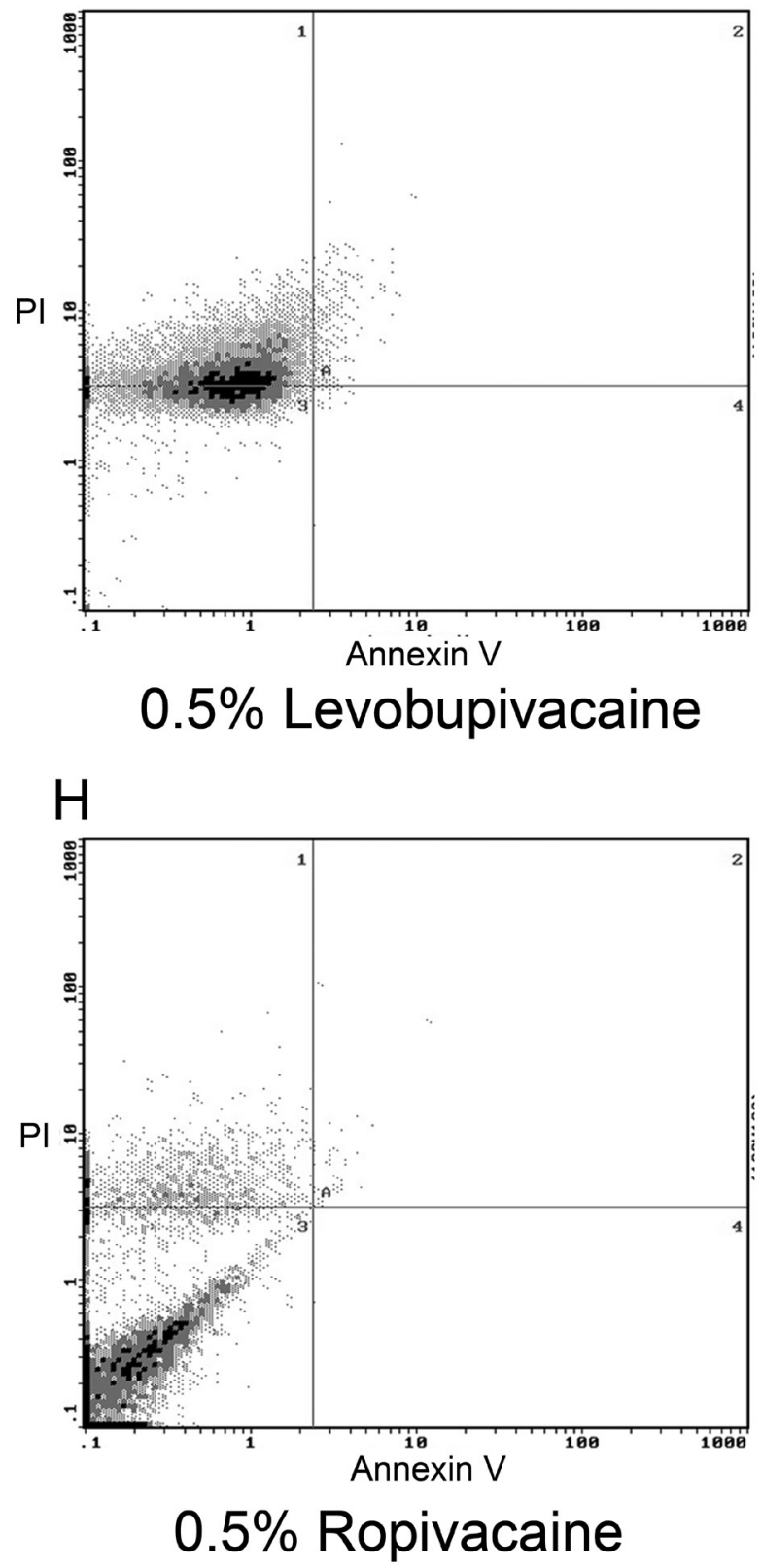

Figure 2E-H. Pattern of CHON-001 cell death after 120 min exposure to local anesthetics as analyzed using a Beckman Coulter Epics XL flow cytometer after staining with annexin V-conjugated FITC and PI. The numbers of necrotic chondrocytes increased after treatment with $0.25 \%$ levobupivacaine $(\mathbf{E}), 0.5 \%$ levobupivacaine $(\mathbf{F})$, while chondrocytes treated with ropivacaine $(\mathbf{G}, \mathbf{H})$ showed no substantial difference in cell viability compared with those treated with PBS vehicle as a control (A). FITC, fluorescein isothiocyanate; PBS, phosphate-buffered saline; PI, propidium iodide

The cytotoxic effects of bupivacaine, levobupivacaine, and ropivacaine have been noted in previous studies in vitro and in vivo $[1,2,6,7]$. We found that the viability of chondrocytes decreased after treatment with $0.25 \%$ or $0.5 \%$ bupivacaine, or $0.5 \%$ levobupivacaine for $60 \mathrm{~min}$, compared with treatment by PBS as a vehicle control. However, treatment with $0.25 \%$ or $0.5 \%$ ropivacaine did not decrease chondrocyte viability at $60 \mathrm{~min}$ when compared with treatment by
PBS. Our findings are similar to those of Piper et al. [2] who reported that ropivacaine was less toxic than bupivacaine or lidocaine on both monolayer cultured cells and intact cartilage after 30-min exposure [2]. Studies by Baker et al. [1] and Breu et al. [8] suggested that chondrocyte damage by local anesthetics was dose and time dependent. Breu et al. [8] demonstrated that exposure to a high concentration $(0.75 \%)$ of ropivacaine decreased chondrocyte viability [8] and suggested that local 


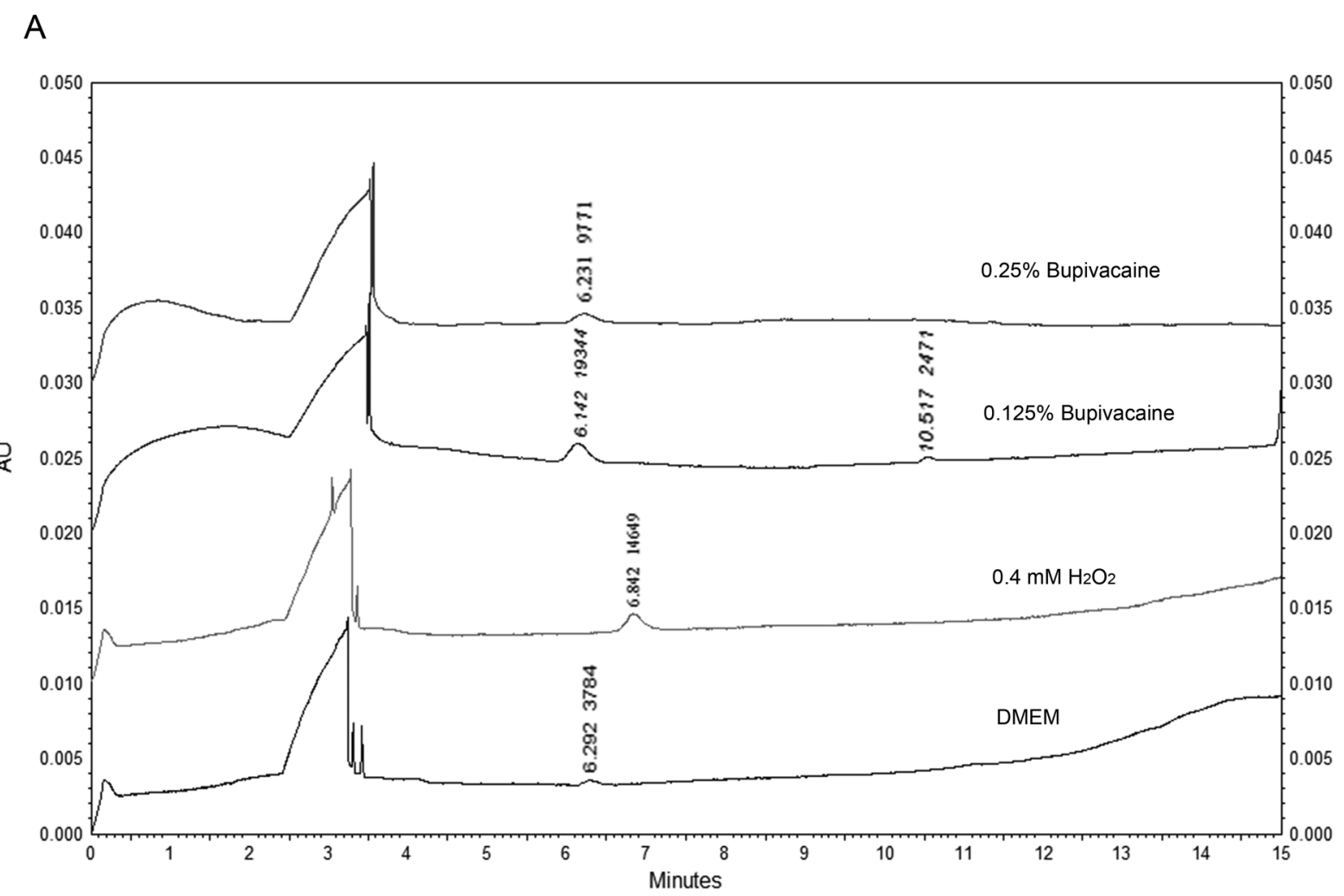

B

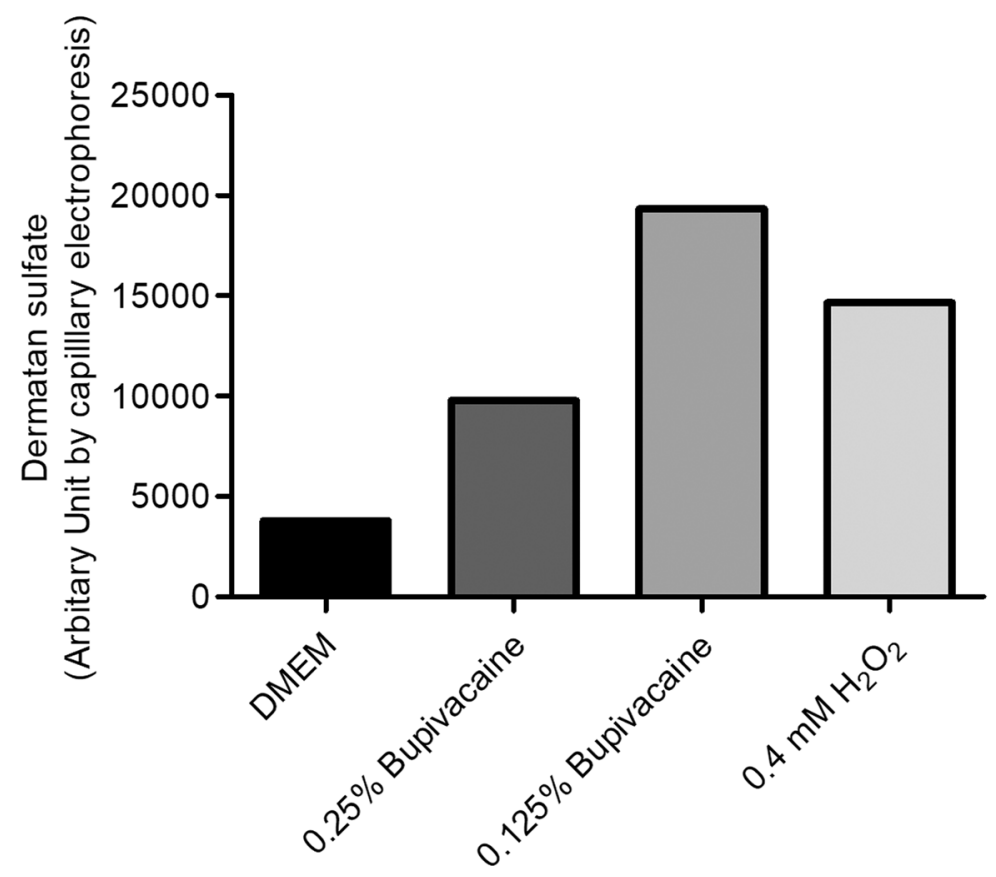

Figure 3. Production of dermatan sulfate by bupivacaine-treated CHON-001 cells as determined by a Beckman Coulter P/ACE MDQ capillary electrophoresis system equipped with a photodiode array detector operating at $195 \mathrm{~nm}$ (AU). (A) Dermatan sulfate production by chondrocytes treated with $0.125 \%$ bupivacaine for $24 \mathrm{~h}$ was greater than production by chondrocytes treated with $0.25 \%$ bupivacaine for $24 \mathrm{~h}$. Labeled peaks correspond to dermatan sulfate showing migration time $(\mathrm{min})$ and relative area under the peak (arbitrary units). Treatments for $24 \mathrm{~h}$ as indicated. (B) Bars show the relative increase in dermatan sulfate production by the chondrocytes after the various treatments including both concentrations of bupivacaine (single measurements). AU, arbitrary units; DMEM, Dulbecco's modified Eagle's medium 
A

$0.25 \%$ Bupivacaine

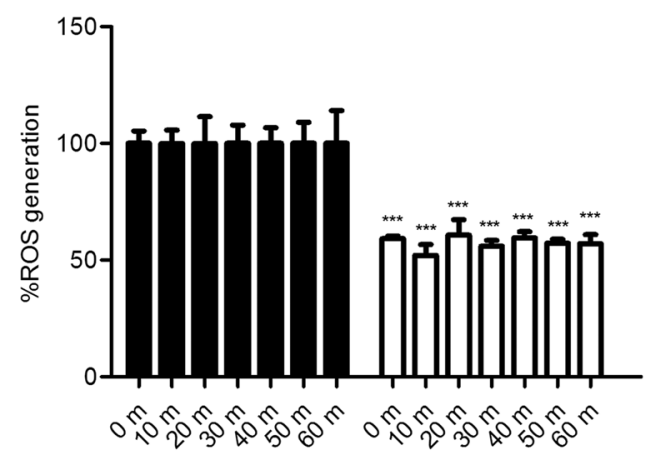

C

$0.25 \%$ Ropivacaine

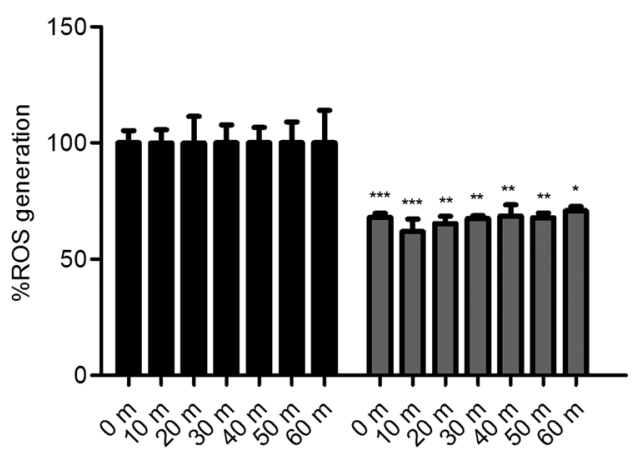

E

$0.25 \%$ Levobupivacaine

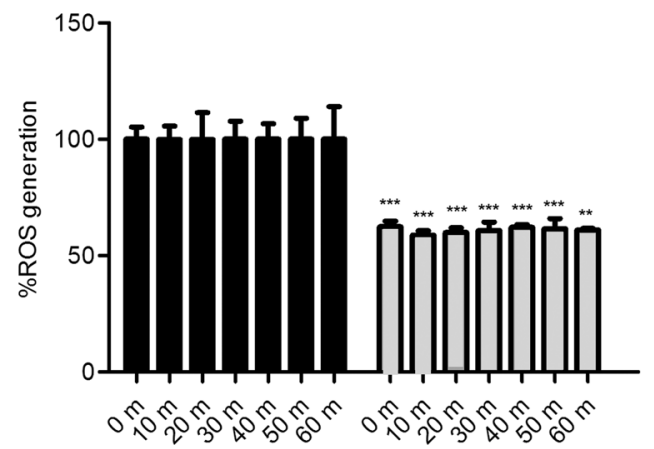

B

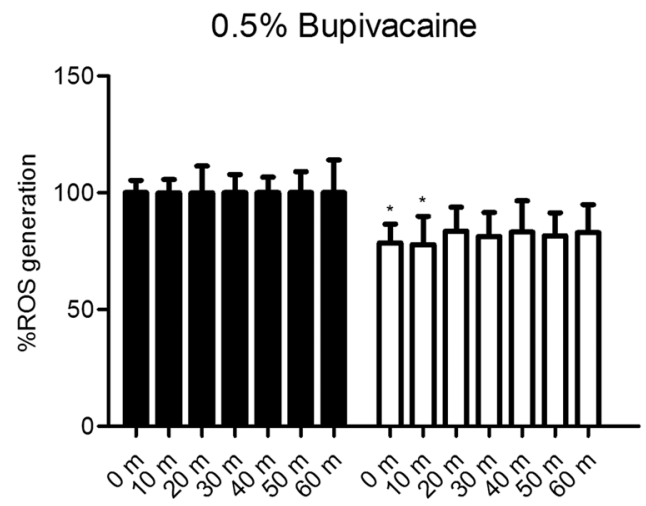

D

$0.5 \%$ Ropivacaine

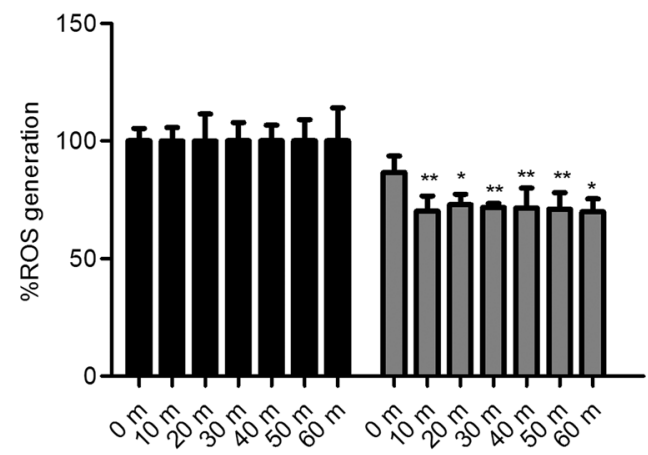

F

$0.5 \%$ Levobupivacaine

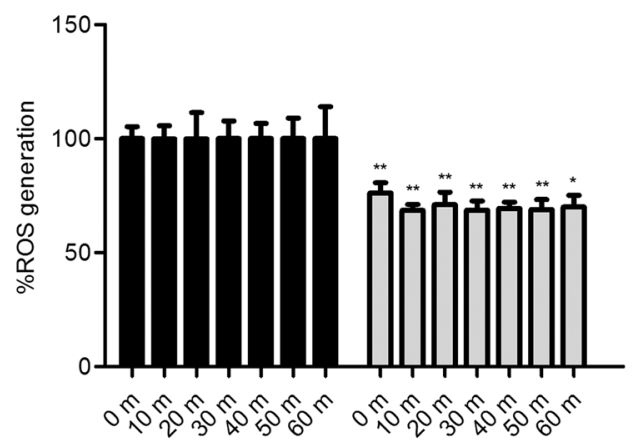

Figure 4. ROS production by $\mathrm{CHON}-001$ cells after exposure to local anesthetics for $0-60 \mathrm{~min}$ as determined by $2^{\prime}, 7^{\prime}$-dichlorodihydrofluorescein diacetate reagent. The chondrocytes were treated with PBS vehicle as a control (black bars), bupivacaine (white bars) $0.25 \%(\mathbf{A})$ and $0.5 \%$ (B), ropivacaine (dark-gray bars) $0.25 \%$ (C) and $0.5 \%$ (D), levobupivacaine (light-gray bars) $0.25 \%$ (E) and $0.5 \%$ (F). The local anesthetics immediately decreased ROS production compared with PBS. ${ }^{*} P<0.05$, ${ }^{* *} P<0.01$, and ${ }^{* * *} P<0.001$; error bars represent standard deviation $\mathrm{m}$, minutes; PBS, phosphate-buffered saline; ROS, reactive oxygen species

anesthetics cause significant chondrotoxicity after a short exposure.

The present study demonstrated that low $(0.25 \%)$ and high $(0.5 \%)$ doses of bupivacaine and a high $(0.5 \%)$ dose of levobupivacaine induced necrotic cell death in chondrocytes after 120 min treatment. The morphology of bupivacainetreated chondrocytes changed from round to elongated. Cytoskeleton disruption and mitochondrial dysfunction may 


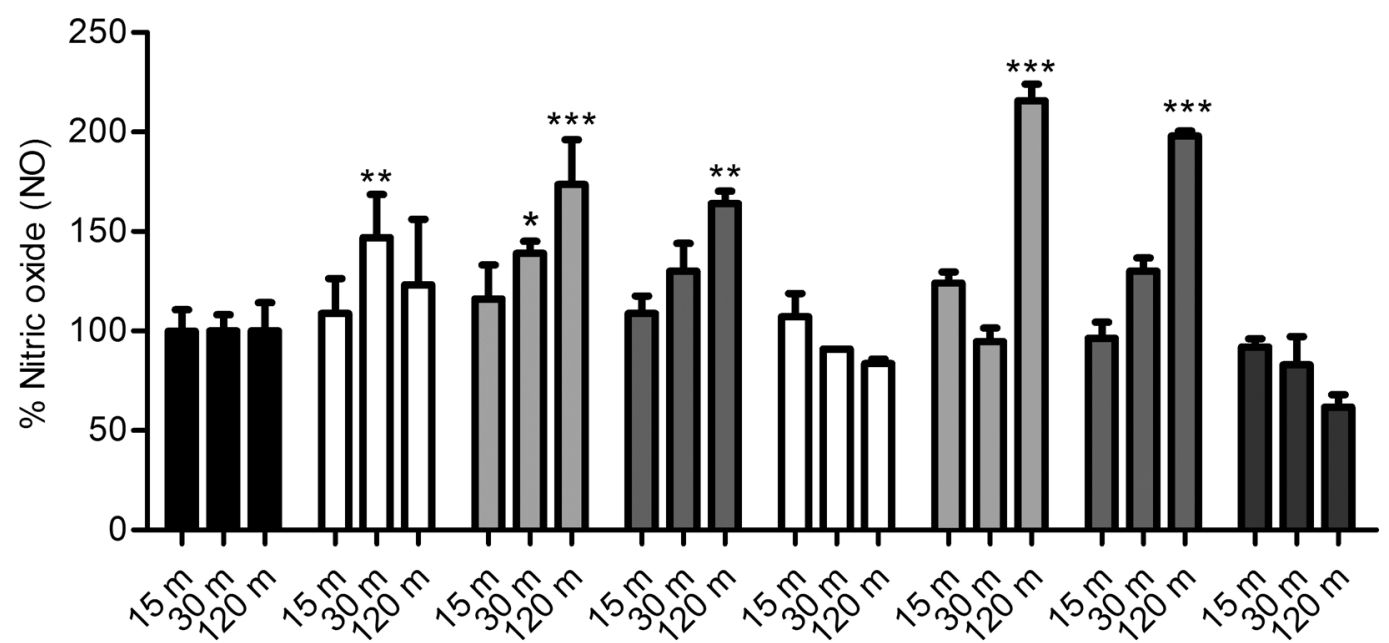

Figure 5. NO production by CHON-001 cells after exposure to local anesthetics for 120 min as measured by using a colorimetric Nitrite/Nitrate Assay kit. The bars represent the mean NO production after treatment with PBS vehicle as a control (black bars), bupivacaine (white bars, $0.25 \%$ left, $0.5 \%$ right), levobupivacaine (light-gray bars, $0.25 \%$ left, $0.5 \%$ right), ropivacaine (medium-gray bars, $0.25 \%$ left, $0.5 \%$ right), and $0.4 \mathrm{mM} \mathrm{H}_{2} \mathrm{O}_{2}$ (dark-gray bars, far right). ${ }^{*} P<0.05,{ }^{* *} P<0.01$, and ${ }^{* * *} P<0.001$; error bars represent standard deviation; $\mathrm{m}$, minutes; NO, nitric oxide

be induced by local anesthetics and may lead to cell death. Varying toxicity has been observed for different durations of exposure to anesthetics. Bupivacaine has an onset of $5 \mathrm{~min}$ and 2-4-h duration of action [9]. Piper et al. [2] found an increase in necrotic cell death of bupivacaine-treated chondrocytes at $24 \mathrm{~h}$, but viability after 24 and $96 \mathrm{~h}$ was not different. These findings suggest that both cell viability and the pattern of cell death are dependent on time of exposure to these anesthetics.

Dermatan sulfate, a GAG, plays a role in cellular proliferation and differentiation, wound healing, anticoagulant, antithrombotic, and anti-inflammatory activities [10]. We found that dermatan sulfate synthesis in chondrocytes treated with a low-dose of bupivacaine was higher than that in chondrocytes treated with a high-dose of bupivacaine. Bupivacaine acts directly on the extracellular matrix, resulting in dermatan sulfate production [11]. Gulihar and Shaunak [12] showed that all local anesthetics tested inhibited proteoglycan metabolism in articular cartilage and that glucosamine was able to reduce the inhibition. Glucosamine produces GAG, which is able to protect the articular cartilage [12]. These findings suggest that chondrocytes remain functional because GAG can protect chondrocyte function after exposure to local anesthetics.

The mechanism of chondrotoxic effect induced by local anesthetics remains unclear. A few studies examined ROS production by chondrocytes treated with various local anesthetics $[13,14]$. Other studies found that local anesthetics play a role in anti-inflammatory effects [15-17]. Hattori et al. demonstrated that different concentrations and types of local anesthetics suppressed free radical production by PMN cells [18]. In chondrocytes, we found an increased production of $\mathrm{NO}$, while ROS decreased after exposure to any of the local anesthetics tested. Previous studies found that NO release is induced by dermatan sulfate and NO produces reactive nitrogen species (RNS) resulting in inflammation [19, 20]. These findings suggest that the local anesthetics may exert a cytotoxic effect on the chondrocyte via an RNS-dependent pathway.

Although the present study supports previous findings of the cytotoxicity of bupivacaine and levobupivacaine in vitro, the mechanisms and pathways involved in the cytotoxic effect of these anesthetics need to be clarified. Limitations include those of using capillary electrophoresis and that we have focused on GAG, dermatan sulfate, chondroitin sulfate, and hyaluronic acid, while there are other types of GAG or extracellular matrix as well as cytoskeleton, which may play important roles in anesthetic cytotoxicity. In the present study of $\mathrm{CHON}-001$ cell line in vitro a few factors can affect cytotoxicity. Hence, further studies of the mechanism of chondrotoxic effects of local anesthetics in vitro and effect of other local anesthetics that are injected intraarticularly are warranted. A clinical study to assess the effect of ropivacaine in articular surgery is also warranted because of its apparently lower toxicity compared with bupivacaine and levobupivacaine.

\section{Conclusions}

Our data support findings of others that chondrotoxicity of the local anesthetics tested in vitro is dependent on time of exposure, concentration, and drug type. Ropivacaine may be safer than bupivacaine or levobupivacaine as an intra-articular analgesic. Chondrotoxicity of anesthetics in vitro may be 
mediated via an RNS-dependent pathway and this warrants further investigation.

Author contributions. PK, SC, and AS contributed substantially to the conception and design of this study. PK, MS-A, SS, VA, and CC contributed substantially to the acquisition of data. PK, SC, MS-A, and AS analyzed and interpreted data. $\mathrm{PK}, \mathrm{SC}, \mathrm{VA}$, and AS drafted the manuscript. PK, SC, MS-A, $\mathrm{SS}$, and AS contributed substantially to its critical revision. All the authors approved the final version submitted for publication and take responsibility for the statement made in the published article.

Acknowledgments. The authors gratefully acknowledge the grant to support a research group in the Ratchadaphiseksomphot Endowment Fund, Chulalongkorn University, and the Ratchadapiseksomphot Fund, Faculty of Medicine, Chulalongkorn University (RA58/051). The present work was presented in part as a poster at the 41st Annual Conference of the Anatomy Association of Thailand (AAT41), 2018 May 23-25, Cha-am, Phetchaburi, Thailand. Pudkrong Kaewpichit, Somrat Charuluxananan, Monpichar Srisa-Art, Sarocha Sisawat, Vitavat Aksornkitti, Chalermsri Chayudsatid, Amorpun Sereemaspun. Cellular responses of human chondrocyte after exposure to bupivacaine, levobupivacaine, or ropivacaine: an in vitro evaluation of cytotoxicity and possible mechanistic study [PP 54, AAT120].

Conflict of interest statement. The authors have each completed and submitted an International Committee of Medical Journal Editors Uniform Disclosure Form for Potential Conflicts of Interest. None of the authors have any potential conflict of interest to disclose.

\section{References}

[1] Baker JF, Walsh PM, Byrne DP, Mulhall KJ. In vitro assessment of human chondrocyte viability after treatment with local anesthetic, magnesium sulphate or normal saline. Knee Surg Sports Traumatol Arthrosc. 2011; 19:1043-6.

[2] Piper SL, Kramer JD, Kim HT, Feeley BT. Effects of local anesthetics on articular cartilage. Am J Sports Med. 2011; 39:2245-53.

[3] Chu CR, Izzo NJ, Coyle CH, Papas NE, Logar A. The in vitro effects of bupivacaine on articular chondrocytes. J Bone Joint Surg Br. 2008; 90:814-20.

[4] Chu CR, Izzo NJ, Papas NE, Fu FH. In vitro exposure to $0.5 \%$ bupivacaine is cytotoxic to bovine articular chondrocytes. Arthroscopy. 2006; 22:693-9.
[5] Piper SL, Kim HT. Comparison of ropivacaine and bupivacaine toxicity in human articular chondrocytes. J Bone Joint Surg Am. 2008; 90:986-91.

[6] Chu CR, Coyle CH, Chu CT, Szczodry M, Seshadri V, Karpie JC, et al. In vivo effects of single intra-articular injection of $0.5 \%$ bupivacaine on articular cartilage. J Bone Joint Surg Am. 2010; 92:599-608.

[7] Gulihar A, Robati S, Twaij H, Salih A, Taylor GJ. Articular cartilage and local anaesthetic: a systematic review of the current literature. J Orthop. 2015; 12:S200-10.

[8] Breu A, Rosenmeier K, Kujat R, Angele P, Zink W. The cytotoxicity of bupivacaine, ropivacaine, and mepivacaine on human chondrocytes and cartilage. Anesth Analg. 2013; 117:514-22.

[9] Collins JB, Song J, Mahabir RC. Onset and duration of intradermal mixtures of bupivacaine and lidocaine with epinephrine. Can J Plast Surg. 2013; 21:51-3.

[10] Shang C, Cai C, Zhao C, Du Y. Synthesis and anti-inflammatory activity of gold-nanoparticle bearing a dermatan sulfate disaccharide analog. Chin Chem Lett. 2018; 29:81-3.

[11] Anz A, Smith MJ, Stoker A, Linville C, Markway H, Branson K, Cook JL. The effect of bupivacaine and morphine in a coculture model of diarthrodial joints. Arthroscopy. 2009; 25:225-31.

[12] Gulihar A, Shaunak S, Novak PL, Vinayakam P, Dhinsa B, Taylor G. Glucosamine reduces the inhibition of proteoglycan metabolism caused by local anaesthetic solution in human articular cartilage: an in vitro study. J Exp Orthop. 2017; 4:37. doi: 10.1186/s40634-0170106-4

[13] Lee Y-J, Kim SA, Lee S-H. Hyaluronan suppresses lidocaineinduced apoptosis of human chondrocytes in vitro by inhibiting the p53-dependent mitochondrial apoptotic pathway. Acta Pharmacol Sin. 2016; 37:664-73.

[14] Tian J, Li Y. Comparative effects of vitamin C on the effects of local anesthetics ropivacaine, bupivacaine, and lidocaine on human chondrocytes. Braz J Anesthesiol. 2016; 66:29-36.

[15] Cassuto J, Sinclair R, Bonderovic M. Anti-inflammatory properties of local anesthetics and their present and potential clinical implications. Acta Anaesthesiol Scand. 2006; 50:265-82.

[16] Hollmann MW, Durieux ME. Local anesthetics and the inflammatory response: a new therapeutic indication? Anesthesiology. 2000; 93:858-75.

[17] Saito T, Yamamoto Y, Feng GG, Kazaoka Y, Fujiwara Y, Kinoshita H. Lidocaine prevents oxidative stress-induced endothelial dysfunction of the systemic artery in rats with intermittent periodontal inflammation. Anesth Analg. 2017; 124:2054-62.

[18] Hattori M, Dohi S, Nozaki M, Niwa M, Shimonaka H. The inhibitory effects of local anesthetics on superoxide generation of neutrophils correlate with their partition coefficients. Anesth Analg. 1997; 84:405-12.

[19] Sharma JN, Al-Omran A, Parvathy SS. Role of nitric oxide in inflammatory diseases. Inflammopharmacology. 2007; 15:252-9.

[20] Simonaro CM, Haskins ME, Schuchman EH. Articular chondrocytes from animals with a dermatan sulfate storage disease undergo a high rate of apoptosis and release nitric oxide and inflammatory cytokines: a possible mechanism underlying degenerative joint disease in the mucopolysaccharidoses. Lab Invest. 2001; 81:1319-28. 